\title{
Human liver stem cells attenuate concanavalin A-induced acute liver injury by modulating myeloid-derived suppressor cells and $\mathrm{CD}^{+} \mathrm{T}$ cells in mice
}

Yanzhen $\mathrm{Bi}^{1 \dagger}$, Jiannan $\mathrm{Li}^{2+}$, Yonghong Yang ${ }^{3}$, Quanyi Wang ${ }^{3}$, Quanquan Wang ${ }^{4}$, Xiaobei Zhang ${ }^{3}$, Guanjun Dong ${ }^{5}$, Yibo Wang ${ }^{3}$, Zhongping Duan ${ }^{1}$, Zhenfeng Shu ${ }^{6}$, Tongjun Liư ${ }^{2}$, Yu Chen ${ }^{1 *}$, Kai Zhang ${ }^{2^{*}}$ and Feng Hong ${ }^{3^{*}}$

\begin{abstract}
Background: Acute liver failure (ALF) is a serious threat to the life of people all over the world. Finding an effective way to manage ALF is important. Human liver stem cells (HLSCS) are early undifferentiated cells that have been implicated in the regeneration and functional reconstruction of the liver. In this study, we aimed to evaluate the protective effects of the HLSC line HYX1 against concanavalin A (ConA)-induced acute liver injury.

Methods: HYX1 cells were characterized by microscopy, functional assays, gene expression, and western blot analyses. We showed that HYX1 cells can differentiate into hepatocytes. We intraperitoneally injected HYX1 cells in mice and administered ConA via caudal vein injection 3, 6, 12, 24, and $48 \mathrm{~h}$ later. The effects of HYX1 cell transplantation were evaluated through blood tests, histology, and flow cytometry.

Results: HYX1 cells reduced the levels of alanine transaminase (ALT), aspartate aminotransferase (AST), and total bilirubin (TBIL) in serum and dramatically decreased the severity of liver injuries. Mechanistically, HYX1 cells promoted myeloid-derived suppressor cell (MDSC) migration into the spleen and liver, while reducing $\mathrm{CD4}^{+}{ }^{\top}$ cell levels in both tissues. In addition, HYX1 cells suppressed the secretion of proinflammatory cytokines, such as tumour necrosis factor-a (TNF-a) and interferon- $\gamma$ (IFN- $\gamma)$, but led to increased interleukin-10 (IL-10) production.
\end{abstract}

Conclusions: These results confirm the efficacy of HLSCs in the prevention of the ConA-induced acute liver injury through modulation of MDSCs and $\mathrm{CD}^{+} \mathrm{T}$ cell migration and cytokine secretion.

Keywords: Acute liver injury, Human liver stem cells, MDSCs, CD4 ${ }^{+} \mathrm{T}$ cells, Concanavalin A

\section{Background}

The liver is the largest internal organ and performs very important functions, such as production of bile, storage of glycogen, and filter of harmful substances, among others. Liver diseases, especially acute liver failure (ALF), seriously threaten the life of people all over the world [1].

\footnotetext{
*Correspondence: chybeyond@163.com; zhangkai0628@126.com; fenghong9508@163.com

${ }^{\dagger}$ Yanzhen Bi and Jiannan Li contributed equally to this work.

'Beijing Artificial Liver Treatment \& Training Center, Beijing Youan Hospital, Captial Medical University, Beijing 100069, People's Republic of China

${ }^{2}$ Department of General Surgery, The Second Hospital of Jilin University, Changchun 130041, People's Republic of China

${ }^{3}$ Institute of Liver Diseases, Affiliated Hospital of Jining Medical University, Jining 272067, People's Republic of China

Full list of author information is available at the end of the article
}

The factors that induce liver damage mainly include over-ingestion of some drugs, excessive drinking, viral infections, and autoimmune responses [2, 3]. ALF is often caused by autoimmune hepatitis and hepatitis $\mathrm{B}$ or $\mathrm{C}$ infection [4]. Concanavalin A (ConA) administration can significantly increase the liver transaminase levels and leads to acute hepatitis $[5,6]$. The ConA-induced acute liver injury model is characterized by liver necrosis, activation of $\mathrm{T}$ lymphocytes, and overexpression of free radicals and pro-inflammatory cytokines $[7,8]$. Therefore, this model is widely accepted for mechanistic studies of ALF.

ALF is characterized by acute onset, rapid progression, and a low survival rate. In addition, it often affects the function of multiple organ systems [4]. Consequently, 
finding an efficient way to treat/improve ALF is necessary and important. Nowadays, orthotopic liver transplantation and artificial liver systems are the usual treatment methods for ALF. Unfortunately, the possibility of liver transplantation is limited by the shortage of liver donors, immunological suppression, and high costs [9]. Stem cells, which can be derived from other tissues and expanded in vitro, have improved prospects for the treatment of many diseases [10, 11]. Liver stem cells (LSCs) are early undifferentiated cells which can proliferate efficiently, differentiate in multiple directions, and self-renew. Furthermore, they can also be involved in the functional regeneration of the liver [12, 13]. With the development of isolation and culture techniques for stem cells, LSCs might provide a new way to treat ALF $[14,15]$.

Myeloid-derived suppressor cells (MDSCs) are heterogeneous immature myeloid cells and characterized by the ability to suppress the proliferation and cytotoxicity of $\mathrm{T}$ cells and induce the expansion of regulatory $\mathrm{T}$ cells (Treg), which play an important role in hepatic protection [16]. The liver has been shown to be a site for MDSC accumulation that is associated with hepatic diseases [17]. Patients infected by hepatic viruses present increased levels of MDSCs in the peripheral blood [18]. Previous studies have also reported that $\mathrm{CD}^{+}{ }^{+} \mathrm{T}$ lymphocytes increased ConA-induced acute liver injury [19, 20]. However, MDSCs can suppress and modulate the phenotype of $\mathrm{CD} 4^{+} \mathrm{T}$ cells [17].

In the present study, we investigated the protective effect of human liver stem cells (HLSCs) against ConA-induced acute liver injury and further analysed their mechanisms of action in this murine model of ALF.

\section{Methods \\ Materials}

Human liver stem cells 1 (HYX1) were provided by Professor Hong Feng. ConA was purchased from Sigma-Aldrich (Shanghai, P. R. China). Dulbecco's modified Eagle medium (DMEM) and foetal bovine serum (FBS) were purchased from Gibco (Gran Island, NY, USA). Indocyanine green (ICG) and periodic acid-schiff (PAS) were purchased from Sigma-Aldrich (Shanghai, P. R. China). Periodic acid and sulfuric acid were purchased from Beijing Chemical Works (Beijing, P. R., China). Paraformaldehyde, dimethyl sulfoxide (DMSO), and hepatocyte growth factor (HGF) were obtained from Beijing Huafeng United Technology Co., Ltd. (Beijing, P. R., China).

\section{Isolation and culture of HYX1 cells}

Human liver specimens (20 to $50 \mathrm{~g}$ ) obtained from Jining Medical University Affiliated Hospital were first perfused in situ for $10 \mathrm{~min}$ with 200 to $300 \mathrm{~mL}$ of EGTA buffer and collagenase type IV (0.05\%) in DMEM until the tissue became soft and showed signs of dissolution. The tissue was minced, and the cell suspension was centrifuged twice at $50 \times g$ for $2 \mathrm{~min}$ at $4{ }^{\circ} \mathrm{C}$. The supernatant was collected and centrifuged at $150 \times g$ for $8 \mathrm{~min}$ at $4^{\circ} \mathrm{C}$. The resultant cell pellet was resuspended in DMEM and centrifuged at $150 \times g$ for $5 \mathrm{~min}$ at $4{ }^{\circ} \mathrm{C}$. Finally, the pelleted cells containing crude HLSCs were suspended in PBS for purification in density gradients made of 50\%, 70\%, and 90\% Percoll (Sigma-Aldrich) and cell suspension. To spread layer by layer from the bottom of the tube, place the cell suspension on the top layer. The preparation was centrifuged at $350 \times g$ for $20 \mathrm{~min}$ at $4{ }^{\circ} \mathrm{C}$. The interface between the $50 \%$ and $70 \%$ Percoll was decanted to a tube and centrifuged at $350 \times$ $\mathrm{g}$ for $5 \mathrm{~min}$. The cell pellet was resuspended in DMEM and centrifuged twice at $1200 \mathrm{rpm}$ for $5 \mathrm{~min}$ at $4{ }^{\circ} \mathrm{C}$.

The purified HLSCs were collected and used for culture in six-well plates in DMEM supplemented with $10 \%$ FBS, $100 \mathrm{U} / \mathrm{ml}$ penicillin, $100 \mu \mathrm{g} / \mathrm{ml}$ streptomycin, $1 \mathrm{mg} / \mathrm{l}$ insulin, and $1 \times 10^{7} \mathrm{~mol} / \mathrm{l}$ dexamethasone. These were cultured for 2-3 weeks with the medium changed twice a week. When colonies became visible, they were encircled with cloning rings and subcultured to an individual well of a six-well plate. The expanded cells were taken for assessment of markers of hepatic stem cells. The human liver stem cells isolated are named HYX1, which can be currently subcultured to 50 generations. The initial batch of HYX1 cells was cultured for 20 days, and the cells were photographed after the 10th passage under a phase-contrast microscope (CKX31, Olympus, Tokyo, Japan). The high-resolution morphology of HYX1 cells was examined by transmission electron microscopy (TEM, JEOL, Tokyo, Japan). Thereafter, cells were transferred to T-75 flasks. At confluence, cells were taken for experiments.

\section{ICG uptake assays}

ICG uptake assays were used to analyse the hepatic function of HYX1 cells. Briefly, HYX1 cells (10th passage) were treated with $1 \mathrm{mg} / \mathrm{ml}$ ICG at $37^{\circ} \mathrm{C}$ for $1 \mathrm{~h}$. The cells were washed twice with phosphate-buffered saline (PBS) and resuspended with DMEM, low glucose $(1000 \mathrm{mg} / \mathrm{L})$ containing $10 \%$ FBS. The cells were then observed under a CKX31 microscope.

\section{PAS staining}

PAS staining was used to estimate the glycogen storage functions of the cells. HYX1 cells (10th passage) were treated with $4 \%$ paraformaldehyde for $10 \mathrm{~min}$, washed with PBS, and air dried. The cells were then dealt with in $1 \%$ periodic acid. Finally, the cells were stained with PAS for $30 \mathrm{~min}$ at room temperature, washed with 
sulfuric acid in PBS, and air dried. The cells were analysed under a CKX31 microscope.

\section{Reverse transcription-polymerase chain reaction (RT-PCR) for HYX1 cells}

RT-PCR was performed to analyse expression of albumin $(A L B)$, alpha fetoprotein $(A F P)$, and cytokeratin $(C K) 7$, $C K 8$, and CK19 in HYX1 cells. Total RNA from HYX1 cells was isolated using a RNAiso kit (Takara, Otsu, Japan). The Moloney murine leukaemia virus reverse transcriptase (M-MLV) was used to synthetize cDNA. The resultant cDNA was then subjected to PCR amplification and separated by electrophoresis; the DNA signals on the gel were imaged. The sequences for the primers are listed in Table 1.

\section{Western blot analysis}

Western blot analysis was performed to analyse the ability of HYX1 cells to differentiate in hepatic cells. HYX1 cells were treated with $2 \%$ DMSO and HGF $(20 \mathrm{ng} / \mathrm{mL})$ for 5 days in $2 \%$ DMSO+HGF group, and the control group was added to an equal volume of PBS for 5 days. Proteins were extracted from HYX1 cells. The protein concentrations were determined using a bicinchoninic acid (BCA) protein assay kit (Beyotime, Nanjing, P. R. China). Equal amounts of proteins were separated by sodium dodecyl sulfate-polyacrylamide gel electrophoresis (SDS-PAGE) and transferred to polyvinylidene difluoride (PVDF) membranes. The membranes were blocked in 5\% skim milk and incubated with primary antibodies against ALB (1:1000 dilution, Abcam, Cambridge, UK) and CK19 (1:1000 dilution, Abcam) and HRP-conjugated secondary antibodies (1:3000; Boster, Wuhan, China). Finally, the immunoreactive signals were detected using an enhanced chemiluminescence (ECL) plus kit (Millipore, Temecula, CA, USA).

\section{Mice and treatment}

Male C57BL/6 mice weighing 22-25g (Jinan Pengyue Laboratory Animal Breeding Co., Ltd., Jinan, China) were maintained according to the National Institutes of Health guidelines for the care and use of animals, approved by the Institutional Animal Care and Use Committee of Jining Medical University Affiliated Hospital, China. Mice were kept in the Animal Care Facility of
Jining Medical University with a $12 \mathrm{~h}$ light-dark cycle at constant temperature. Mice had free access to tap water during the study period.

In this study, 66 mice were randomly divided into three groups: normal $(n=6)$, ConA (labelled as $\mathrm{M}, n=$ 30 ), and HYX1+ConA (labelled as T, $n=30$ ). In the normal group, mice were injected with $200 \mu \mathrm{L}$ PBS via tail vein. In the $M$ group, mice were injected with $12 \mathrm{mg} / \mathrm{kg}$ ConA via tail vein after $200 \mu \mathrm{L}$ PBS were administered via intraperitoneal (IP) injection. In the $\mathrm{T}$ group, mice were injected with $2.0 \times 10^{6}$ HYX1 cells (10th passage) via IP injection before treatment with ConA. Both the $M$ and $\mathrm{T}$ groups were further divided into subgroups, 1 through 5, injected with PBS (group M) or HYX1 cells (group T) 3, 6, 12, 24, $48 \mathrm{~h}$ before treatment with ConA (Fig. 1a). For the $\mathrm{M}$ and $\mathrm{T}$ groups, $24 \mathrm{~h}$ post-ConA injection, blood was collected and the mice were sacrificed for liver sample collection (Fig. 1a). For normal group, blood serum and liver specimens were collected $24 \mathrm{~h}$ post-PBS injection. Blood serum was evaluated for biochemical parameters, and liver tissues were analysed for histopathology. We found that HYX1 cell injection 6 or $12 \mathrm{~h}$ before treatment with ConA (T2 or T3 groups) had the best effect against acute liver injury [21]. Therefore, based on the results from the M3 and T3 subgroups, we established two more groups called M3+ and T3+ injected with PBS or HYX1 cells, respectively, $12 \mathrm{~h}$ before treatment with ConA; each group contained five different subgroups $(n=6)$ named M3 or T3 $+1,+$ $2,+3,+4$, and +5 , in which mice were sacrificed 3,6 , 12, 24, and $48 \mathrm{~h}$, respectively, after ConA injection (Fig. 1b). At each time point post-ConA injection, blood was collected, and the mice were sacrificed for spleen and liver sample collection. Blood serum was evaluated for biochemical parameters and inflammatory factors. Liver and spleen samples were analysed for histopathology and potential changes of the MDSCs and $\mathrm{CD} 4^{+} \mathrm{T}$ cell populations.

\section{Blood serum analysis}

Before the animals were sacrificed, blood was collected from the orbital plexus and then centrifuged at 3000 $\mathrm{rpm}$ for $10 \mathrm{~min}$ to obtain the serum. The levels of alanine transaminase (ALT), aspartate transaminase (AST), and total bilirubin (TBIL) were detected by

Table 1 RT-PCR primer sequences

\begin{tabular}{lll}
\hline Gene & Forward primer & Reverse primer \\
\hline AFP & 5'-GCAAAGCTGAAAATGCAGTTGA-3' & 5'-GGAAAGTTCGGGTCCCAAAA-3' \\
CK7 & 5'-TGAATGATGAGATCAACTTCCTCAG-3' & 5'-TGTCGGAGATCTGGGACTGC-3' \\
CK8 & 5'-TCATAGACAAGGTACGGTTCC-3' & 5'-GCCTAAGGTTGTTGATGTAGC-3' $^{\prime}$ \\
CK19 & 5'-TCGACAACGCCCGTCTG-3' & 5'-CCACGCTCATGCGCAG-3' $^{\prime}$ \\
ALB & 5'-CTGAGCAAAGGCAATCAACA-3' & 5'-CACAGTCTGCTGAGGTGGA-3' \\
\hline
\end{tabular}




\section{a Groups}

\section{Procedures}

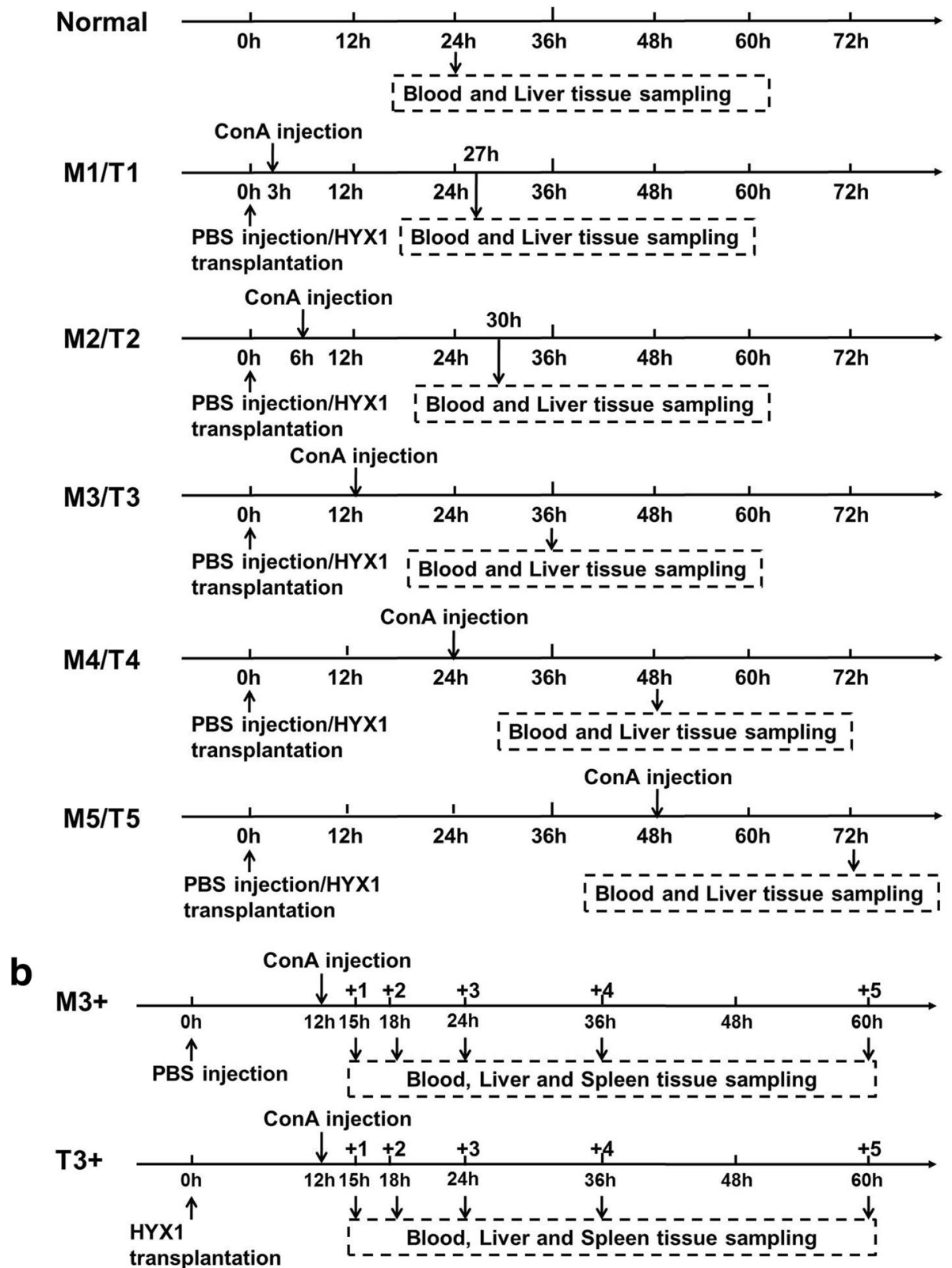

Fig. 1 Schematic outline of the experimental groups. a Initial grouping of the mice; mice were sacrificed for sample collection $24 \mathrm{~h}$ after ConA treatment. $\mathbf{b}$ In the $+1,2,3,4$, and 5 groups, mice were sacrificed for sample collection 3, 6, 12, 24, and 48 h, respectively, after ConA injection

using an automated analyser (AU5800, Beckman Coulter, Brea, CA, USA).

\section{Flow cytometry analysis}

For flow cytometry, fluorescently labelled antibodies against MDSCs (defined as CD11b+ Gr-1+ cells) and CD4 markers were obtained from eBioscience (San Diego, CA, USA) and used at a 1:100 dilution. Cell suspensions from the liver and spleen were prepared after the animals were sacrificed. For phenotype staining, cells were washed twice with PBS containing 1\% FBS and $0.1 \%$ $\mathrm{NaN}_{3}$. The cells were then incubated for $60 \mathrm{~min}$ at $4{ }^{\circ} \mathrm{C}$ with the following anti-mouse antibodies according to standard procedures: CD11b-fluorescein isothiocyanate (FITC), Gr-1-allophycocyanin (APC), CD3-FITC, and CD8a-PerCP Cyanine5.5. After two washes with PBS, 
the cells were analysed using a FACSCalibur (Becton Dickinson, San Jose, CA, USA). An isotype control was used for each antibody.

\section{Haematoxylin and eosin staining}

The liver tissues were collected at the same location as the flow cytometry samples, rinsed with PBS, fixed with $4 \%$ formalin, and embedded in paraffin. The samples were then sectioned and stained with haematoxylin and eosin (H\&E).

\section{Quantitative PCR (qPCR) analysis of mouse liver samples}

Total RNA was extracted from $30 \mathrm{mg}$ of mouse liver. The RNA $(5 \mu \mathrm{g})$ was reverse transcribed into cDNA as described above and analysed in triplicate by qPCR using a SYBR green qPCR Master Mix (Annoron, Roche, Beijing, China) on the LightCycler 480 Real-Time PCR System (Annoron, Roche, Beijing, China). The sequences of the qPCR primers are listed in Table 2.

\section{Enzyme-linked immunosorbent assay (ELISA)}

Mouse serum samples were collected from the orbital plexus. The levels of TNF- $\alpha$, IFN- $\gamma$, and IL-10 were detected by using ELISA kits according to the manufacturer's instructions (R\&D Systems, Minneapolis, MN, USA).

\section{Statistical analysis}

All results were presented as the mean \pm standard error of mean (SEM) and analysed using the SPSS 13.0 statistical software (IBM, Armonk, NY, USA). The differences between each group were compared by one-way analysis of variance (ANOVA) or Kruskal-Wallis test as appropriate. $P<0.05$ (*) indicated statistical significance; $P<0.01(* *)$ and $P<0.001\left(^{* * *)}\right.$ indicated high statistical significance.

\section{Results}

Morphologic characterization of HYX1 cells

During the first 20 days of culture, HYX1 cells that were attached on culture plates appeared small and round, forming colonies (Fig. 2a). In subsequent subculture, the cells looked enlarged and more flattened, resembling

Table 2 Quantitative PCR primer sequences

\begin{tabular}{llc}
\hline Gene & Sequence (5'-3') & Product (bp) \\
\hline GAPDH & F: CTAGAGAGCTGACAGTGGGTAT & 49 \\
TNF- $a$ & R: AGACGACCAATGCGTCCAAA & \\
& F: CCCTCACACTCAGATCATCTTCT & 61 \\
IFN- $y$ & R: GCTACGACGTGGGCTACAG & \\
& F: TCGGTAACTGACTTGAATGTCCA & 93 \\
IL-10 & R: TCGCTTCCCTGTITAGCTGC & \\
& F: TCAAGGCGCATGTGAACTCC & \\
\hline
\end{tabular}

hepatocytes (Fig. 2b). TEM examination showed that HYX1 cells were small, oval, and mostly occupied by oval nuclei, with large nucleus-to-cytoplasm ratios. The major organelles, such as the mitochondria and ribosomes, were underdeveloped, resembling the characteristics of undifferentiated cells (Fig. 2c). ICG uptake assays showed pale green ICG-positive cells (Fig. 2d) and PAS staining showed that a large number of positive cells with cytoplasm appeared red (Fig. 2e). This indicated that HYX1 cells possessed the functions of normal liver cells.

Next, we evaluated the expression of hepatocyte and biliary epithelial cell markers by HYX1 cells. RT-PCR showed that these cells expressed the hepatocyte marker genes $A L B, C K 8$, and AFP (though the expression of the latter was weak), as well as the cholangiocyte marker genes CK7 and CK19 (Fig. 2h). Western blots demonstrate expression of albumin and CK19 by HYX1 cells. Upon exposure to DMSO and HGF, the expression of albumin was elevated, while that of CK19 was lost (Fig. 2f, g). This indicates that HYX1 can be induced into hepatocytes.

\section{Biochemical analysis after HYX1 cell transplantation}

To evaluate the therapeutic potential of HYX1 cell transplantation against ConA-induced acute liver injury, the serum levels of ALT, AST, and TBIL were analysed in the ConA and HYX1 + ConA groups. In a previous paper, we have already reported that the best protection against acute liver injury in mice is achieved when ConA was injected 6 or $12 \mathrm{~h}$ after intraperitoneal transplantation of HYX1 cells [21] (Additional file 1: Figure S1). In Fig. 3, further research indicated that significantly lower levels of ALT, AST, and TBIL were observed 12 and $24 \mathrm{~h}$ after HYX1 transplantation in the T3+ group compared with the M3+ group. The levels of ALT, AST, and TBIL in the T3+ group were the highest at $6 \mathrm{~h}$, and quickly dropped to normal levels by $24 \mathrm{~h}$. In contrast, the M3+ group levels peaked at 12 (TBIL) or 24 (ALT and AST) $\mathrm{h}$, and gradually decreased to normal by $48 \mathrm{~h}$. These results suggest that HYX1 intraperitoneal transplantation has a protective effect on acute liver injury.

\section{Histopathology after HYX1 cell transplantation}

H\&E staining showed no signs of liver cell degeneration, necrosis, or inflammatory infiltration in the normal group (Fig. 4). In the M groups, hepatocellular congestion and oedema were obvious and the liver parenchyma and bile ducts were infiltrated with inflammatory cells. In addition, the hepatic lobule was damaged and large areas of necrosis were observed (Fig. 4). On the other hand, liver injury was not obvious in the T1, T2, or T3 groups and only mild inflammatory cell infiltration was observed in the T2 and T3 groups. In the T1 and T4 groups, hepatic cell congestion, oedema, inflammatory 


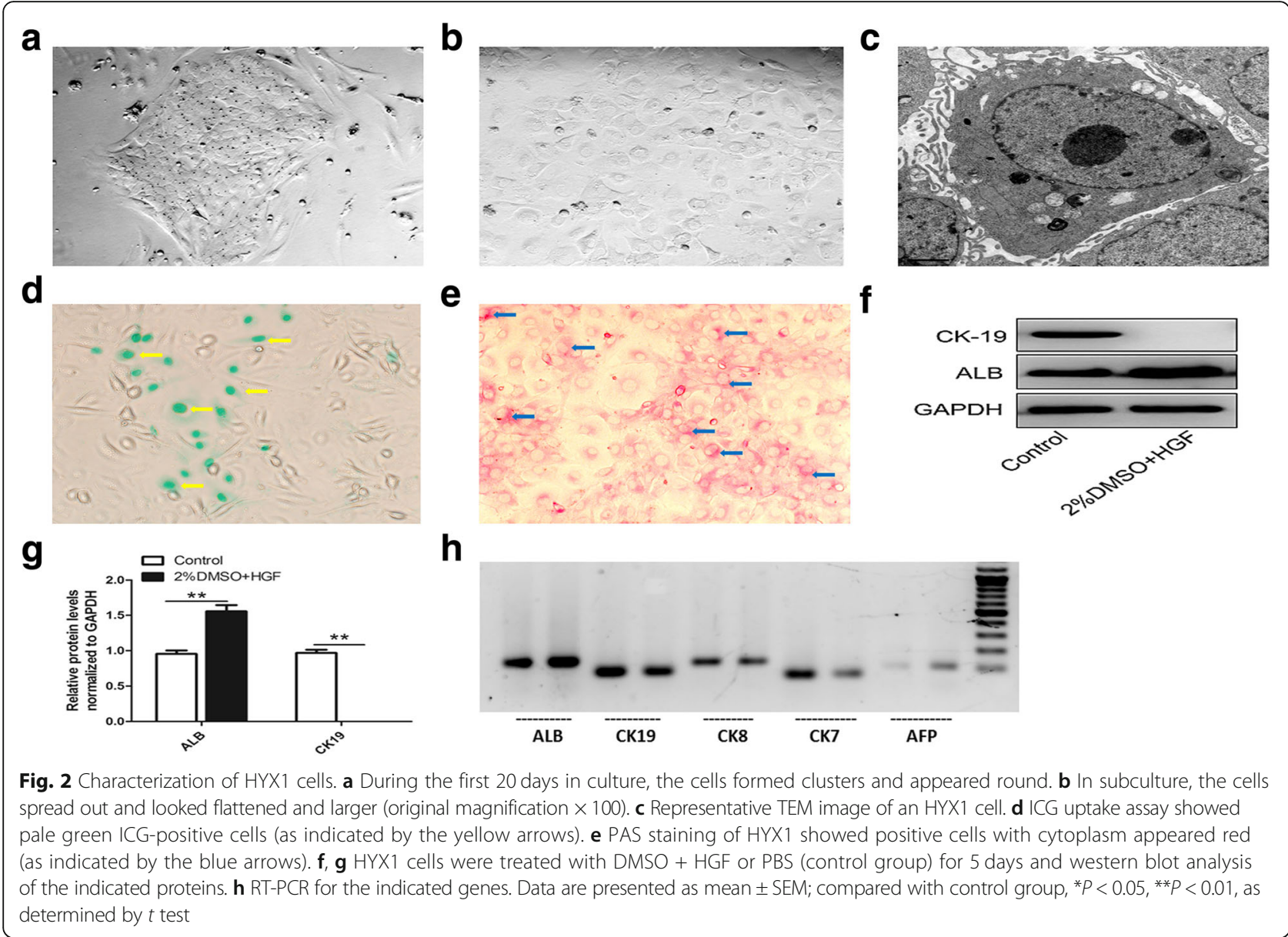

infiltration, punctate, and flaky necrosis were markedly relieved compared to the M4 group. However, the T5 group showed hepatocellular congestion and oedema, considerable inflammation, and a small amount of punctate and flaky necrosis, while the basic hepatic lobules remained intact. These changes were consistent with the differences observed in the biochemical data (Additional file 1: Figure S1).

Furthermore, we found that hepatocyte necrosis worsened from 3 to $48 \mathrm{~h}$ in the M3+ group (Fig. 5). Although the levels of ALT, AST, and TBIL had dropped to normal at $48 \mathrm{~h}$ (Fig. 3), H\&E staining showed that the necrosis was still evident in the M3+ group at this time point (Fig. 5). As shown in Fig. 5, there were only mild hepatocellular congestion, oedema, and inflammatory infiltration in the T3+ group. These results indicate that HYX1 intraperitoneal transplantation is protective against ConA-induced acute liver injury.

\section{Modulation of MDSCs and CD4 ${ }^{+}$T cells after HYX1 cell transplantation}

To evaluate the mechanisms of HYX1 cells against ConAinduced acute liver injury, we performed flow cytometry analyses of MDSCs and $\mathrm{CD}^{+} \mathrm{T}$ cells in liver and spleen
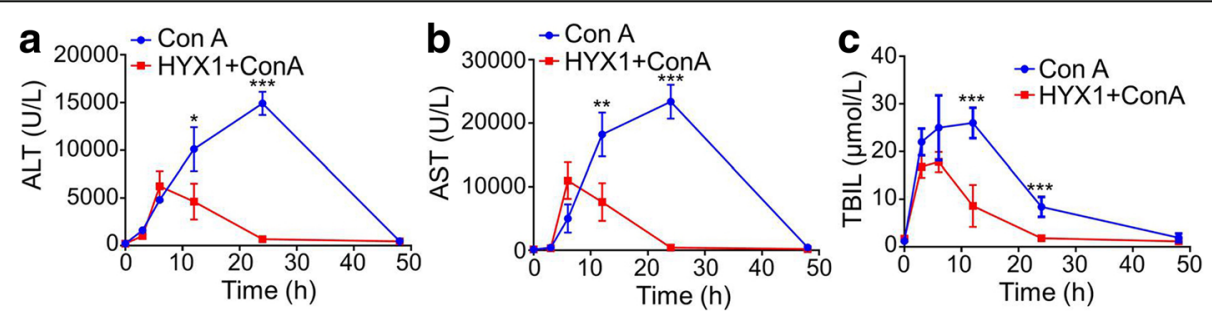

Fig. 3 Biochemical evaluation of a ALT, b AST, and c TBIL in serum from the M3+ (blue) and T3+ (red) mice. Data are presented as mean \pm SEM $\left(n=6\right.$; $\left.{ }^{*} P<0.05,{ }^{*} P<0.01,{ }^{* * *} P<0.001\right)$, as determined by ANOVA tests or $t$ test; ns denotes $P>0.05$ 

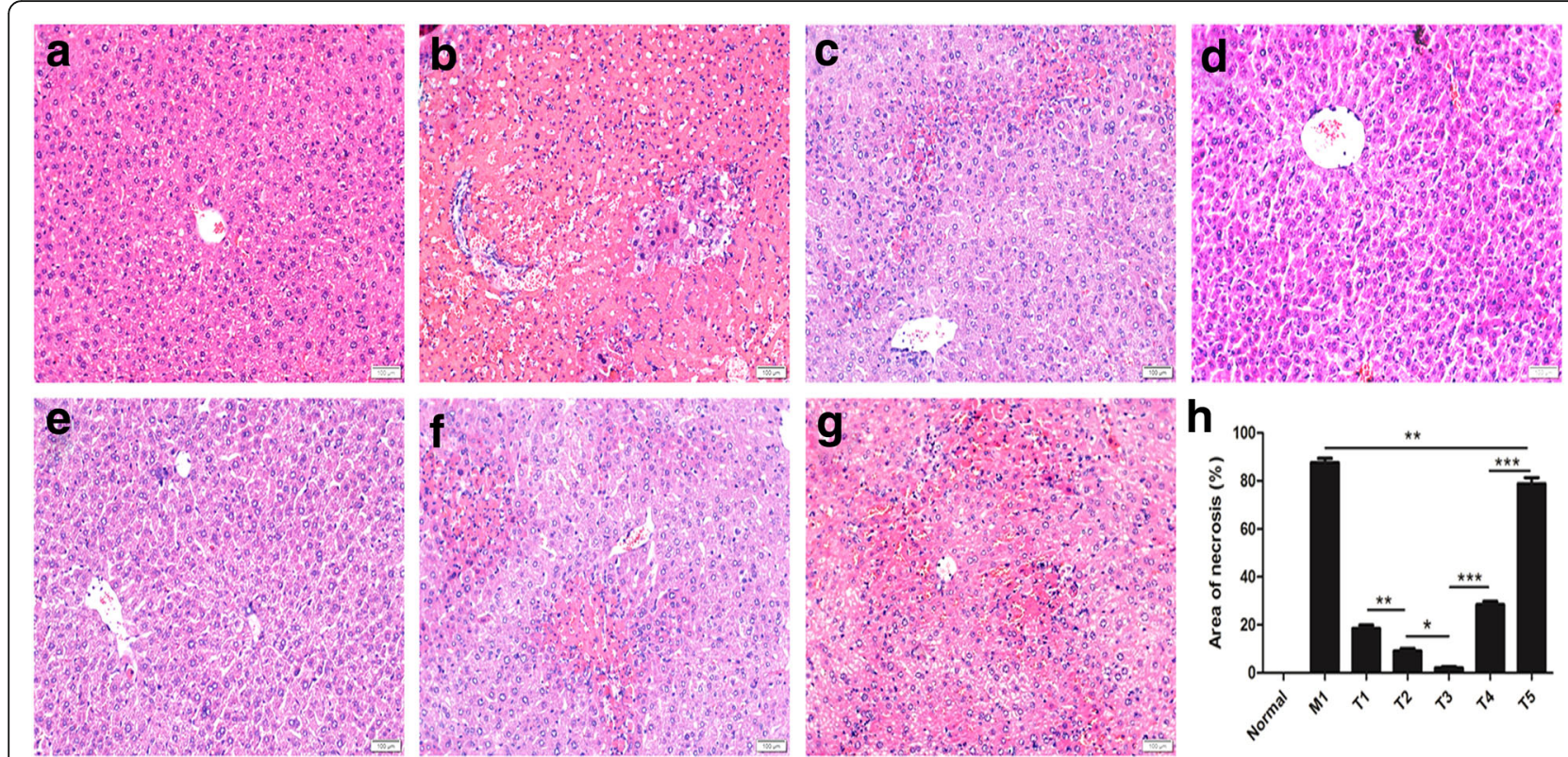

Fig. 4 Representative images of haematoxylin and eosin staining of the liver and quantitation of necrosis area in the normal, $M$, and $T$ groups. a Normal group; b M1 group; $\mathbf{c}-\mathbf{g}$ T1, T2, T3, T4, and T5 groups; $\mathbf{h}$ necrosis area of each group. Data are presented as mean \pm SEM $\left(n=6\right.$; ${ }^{*} P<0.05$, ${ }^{* *} P<0.01,{ }^{* *} P<0.001$ ), as determined by ANOVA tests; ns denotes $P>0.05$. Scale bar $=100 \mu \mathrm{m}$

samples from the different groups (Fig. 6). The percentage of MDSCs and $\mathrm{CD}_{4}^{+} \mathrm{T}$ cells increased immediately after ConA injection in both the liver and spleen. The percentage of $\mathrm{CD}^{+} \mathrm{T}$ cells increased at $3 \mathrm{~h}$ and then decreased with the increased percentage of MDSCs in both the liver and spleen $(\mathrm{M} 3+1, \mathrm{~T} 3+1)$. At $6 \mathrm{~h}$, the percentage of MDSCs reached its peak, while the percentage of $\mathrm{CD} 4^{+} \mathrm{T}$ cells dropped to its lowest level in both tissues $(\mathrm{M} 3+2, \mathrm{~T} 3+2)$. Then, the percentage of MDSCs decreased gradually and dropped to its lowest levels at $24 \mathrm{~h}$, while the percentage of $\mathrm{CD}^{+}{ }^{+} \mathrm{T}$ cells increased gradually and reached its peak at the same time

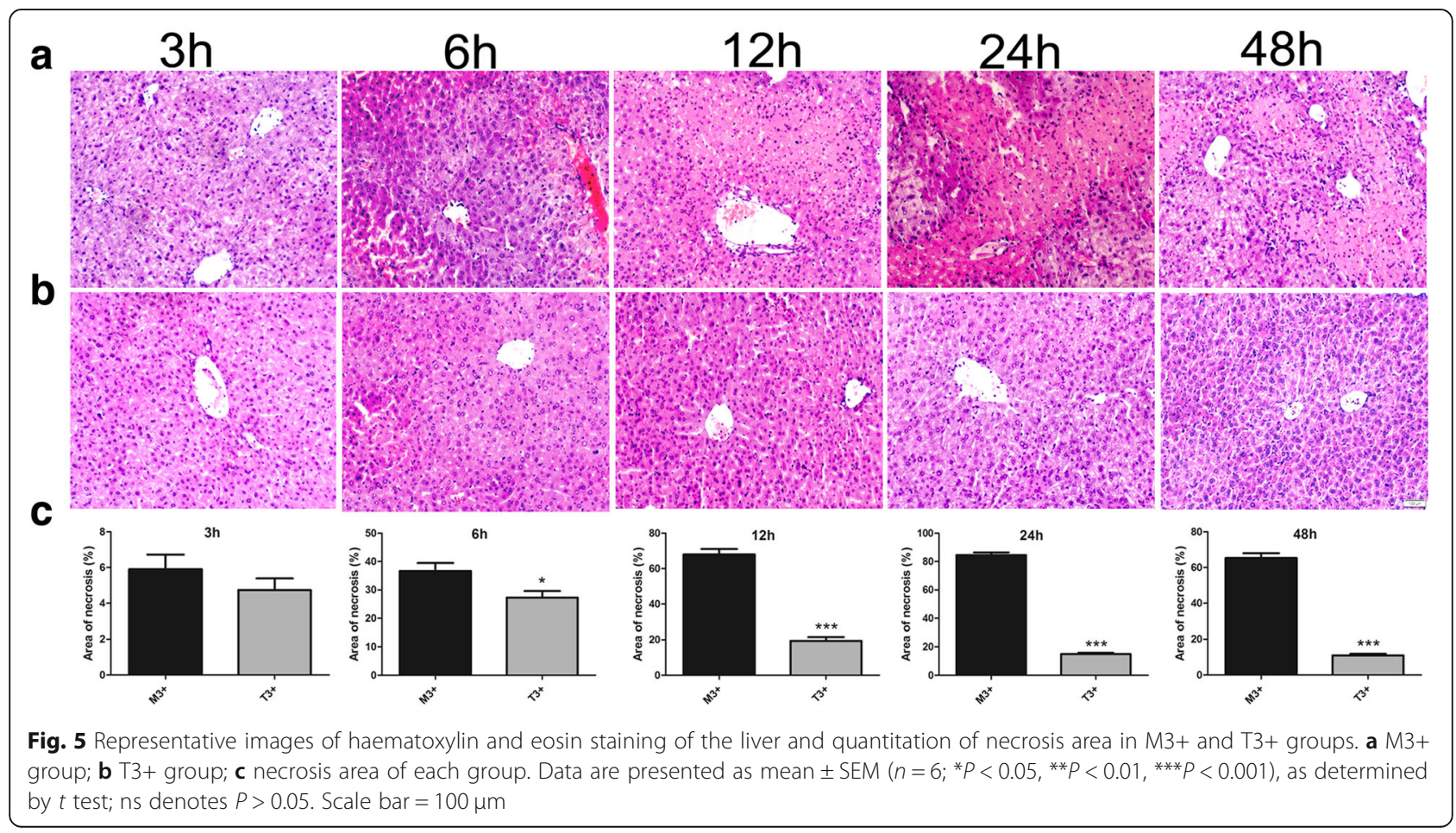



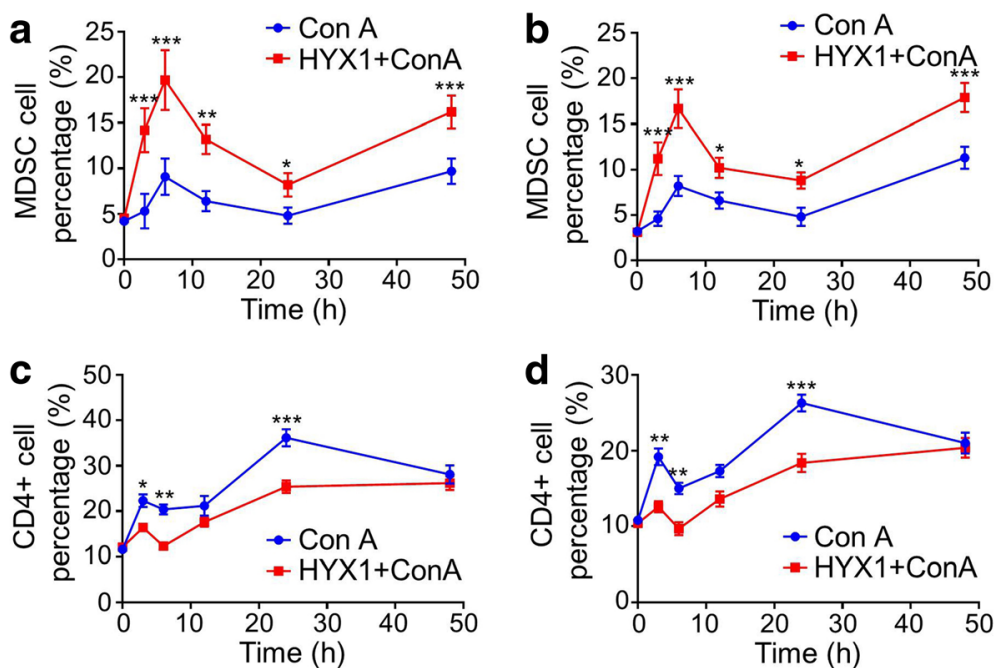

Fig. 6 Changes in MDSCs $(\mathbf{a}, \mathbf{b})$ and $C D 4^{+} T$ cell levels $(\mathbf{c}, \mathbf{d})$ in the liver $(\mathbf{a}, \mathbf{c})$ and spleen $(\mathbf{b}, \mathbf{d})$ in M3+ and T3+ groups of mice. Data are presented as mean $\pm \operatorname{SEM}(n=6) ;{ }^{*} P<0.05,{ }^{* *} P<0.01$, ${ }^{* *} P<0.001$, as determined by ANOVA tests or $t$ test; $n$ ns denotes $P>0.05$

in both the liver and spleen $(\mathrm{M} 3+3, \mathrm{M} 3+4$ and $\mathrm{T} 3+3$, $\mathrm{T} 3+4)$. After $24 \mathrm{~h}$ after ConA injection, the percentage of MDSCs gradually increased again, accompanied by a continuous decline in the percentage of $\mathrm{CD} 4^{+} \mathrm{T}$ cells in both the liver and spleen $(\mathrm{M} 3+4, \mathrm{M} 3+5$ and $\mathrm{T} 3+4, \mathrm{~T} 3$ $+5)$. Interestingly, the fluctuations in the percentage of $\mathrm{CD}^{+}{ }^{+} \mathrm{T}$ cells were consistent with the levels of serum ALT, AST, and TBIL, which were contrary to the dynamics of MDSC presence from 3 to $48 \mathrm{~h}$ post-ConA injection (Figs. 3 and 6).

During the treatment process of HYX1 cells against ConA-induced acute liver injury, the percentages of MDSCs were significantly higher in the HYX1 + ConA group mice $(\mathrm{T} 3+)$ than in the ConA group mice $(\mathrm{M} 3+)$ from 3 to $48 \mathrm{~h}(P<0.05)$, while, conversely, the percentages of $\mathrm{CD} 4^{+} \mathrm{T}$ cells were significantly lower in HYX1 + ConA group mice $(\mathrm{T} 3+1, \mathrm{~T} 3+2, \mathrm{~T} 3+4)$ than in ConA group mice $(\mathrm{M} 3+1, \mathrm{M} 3+2, \mathrm{M} 3+4)$ for both the liver and spleen from 3 to $24 \mathrm{~h}(P<0.05$; Fig. 6$)$.

\section{Detection of TNF-a, IFN- - , and IL-10 serum protein levels by ELISA after HYX1 cell transplantation}

Next, we investigated the levels of pro- and antiinflammatory cytokines in the serum of the different groups of mice. The levels of serum TNF- $\alpha(\mathrm{T} 3+1, \mathrm{~T} 3$ +2 , and $\mathrm{T} 3+3)$ and IFN- $\gamma(\mathrm{T} 3+3$ and $\mathrm{T} 3+4)$ of mice in the $\mathrm{T} 3+$ groups were significantly lower than those in the M3+ groups $(P<0.05$; Fig. $7 \mathrm{a}, \mathrm{b})$, while the levels of IL-10 in the T3+2 group mice were significantly
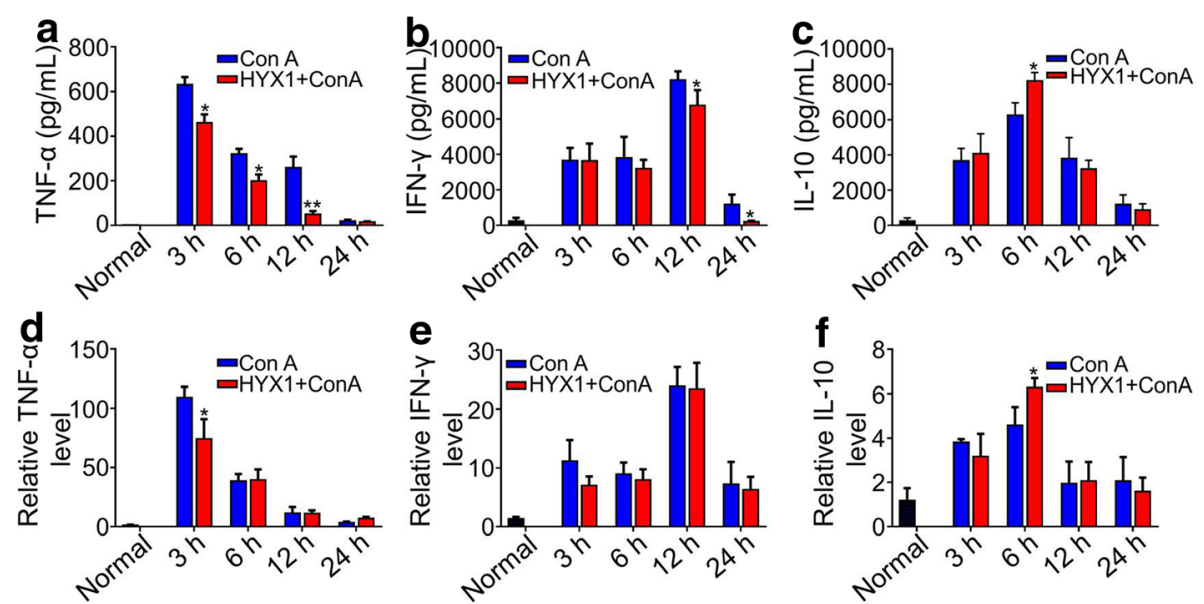

Fig. 7 The protein levels of TNF-a, IFN- - , and IL-10 in serum were quantified by ELISA (a-c) in the M3+ and T3+ groups of mice. The mRNA levels of TNF-a, IFN- $\gamma$, and IL-10 in the liver were quantified by GPCR (d- $\mathbf{f})$ in groups of M3+ and T3+ mice. Data are presented as mean \pm SEM $(n=6) ;{ }^{*} P<0.05,{ }^{* *} P<0.01$, as determined by ANOVA tests; ns denotes $P>0.05$ 
higher at $6 \mathrm{~h}$ compared to those of the $\mathrm{M} 3+2$ group mice $(P<0.05$; Fig. $7 \mathrm{c})$.

\section{Detection of TNF- $a$, IFN- $\gamma$, and IL-10 mRNA levels in liver by qPCR after HYX1 cell transplantation}

We further investigated the effect of HYX1 cells on the expression of TNF- $\alpha, I F N-\gamma$, and $I L-10$ in liver. The results showed that levels of $T N F-\alpha$ decreased significantly in the T3+1 group compared to the M $3+1$ group $(P<$ 0.05; Fig. $7 \mathrm{~d})$. Compared with the $\mathrm{M} 3+$ groups $(\mathrm{M} 3+1$, $\mathrm{M} 3+2, \mathrm{M} 3+4)$, the levels of $I F N-\gamma$ in the liver were also reduced in the $\mathrm{T} 3+$ groups $(\mathrm{T} 3+1, \mathrm{~T} 3+2, \mathrm{~T} 3+4)$, but the differences were not statistically significant $(P>0.05$; Fig. 7e). Furthermore, the levels of $I L-10$ in the $\mathrm{T} 3+2$ group were significantly higher than those of the $\mathrm{M} 3+2$ group $(P<0.05$; Fig. $7 f)$.

\section{Discussion}

ALF, often caused by chronic hepatitis and autoimmune responses, seriously endangers people's lives. Even though medical advances have improved the health of patients with ALF, finding efficient treatment methods for this disorder is still a substantial challenge. In the present study, we used ConA to induce acute liver injury in mice. ConA is a lectin purified from Canavalia ensiformis [22]. The ConA-induced acute liver injury mouse model is the most commonly used animal model for the study of ALF. ConA can specifically activate $\mathrm{CD} 4^{+} \mathrm{T}$ cells and natural killer $\mathrm{T}$ lymphocytes, which can lead to acute liver injury [23]. Different laboratories use different dosages of ConA to induce the acute liver injury [8, 24, 25]. Therefore, we first investigated the appropriate ConA dose for this animal model in our facilities. We found that when $12 \mathrm{mg} / \mathrm{kg}$ ConA were used, the liver injury was serious and obvious and there was no animal death at 24 $\mathrm{h}$ post-ConA injection.

Nowadays, LSCs have gained increased attention for the treatment of liver injury due to their specific functional properties, which are similar to those of normal liver cells, and their ability to be expanded and cultured in large quantities in vitro. In addition, LSC treatment has many other advantages, such as low cost and mild immune rejection responses. Our group have successfully isolated human liver stem cells from liver tissues and developed a cell line (HYX1) which can be stably passed up to 50 generations [21].

In this study, we show that HYX1 cells cultured have the properties of hepatocytes. TEM examination demonstrated the undifferentiated characteristics of HYX1 cells, including large nucleus-to-cytoplasm ratio and underdeveloped organelles. ICG is a water soluble anionic complex that is uptaken by liver cells only and is used to evaluate hepatocyte function [26]. Hepatocytes are very important for glycogen storage and synthesis in vivo. Positive PAS staining is another marker of hepatocyte function. We found that HYX1 cells were stained by ICG and PAS, indicating that they had at least some of the functions of hepatocytes. Furthermore, the RT-PCR results showed that HYX1 express markers of both hepatocytes and bile duct epithelial cells. When HYX1 cells were treated with $2 \%$ DMSO and HGF, the expression of ALB was increased and the expression of CK19 was markedly reduced. This indicated that HYX1 cells could be induced by DMSO and HGF to differentiate into hepatocytes.

We have previously conducted an experiment to study the changes caused by HYX1 transplantation alone. We found that there was almost no changes in serum ALT, AST and TBIL in mice $24 \mathrm{~h}, 48 \mathrm{~h}$, and $72 \mathrm{~h}$ after HYX1 transplantation alone, compared with normal mice (Additional file 1: Figure S4). The results of present study showed that HYX1 cell transplantation provides a protective effect against ConA-induced acute liver injury. Biochemical analyses indicated that HYX1 treatment reduced the serum levels of ALT, AST, and TBIL, especially when HYX1 cells were transplanted 6 or $12 \mathrm{~h}$ before treatment with ConA. However, when HYX1 cells were transplanted $48 \mathrm{~h}$ before ConA treatment, we did not observe a liver-protective effect. Histological examination also revealed that the liver injuries were mild in the T2, T3, and T4 groups (especially in T2 and T3). The results of our study are similar to those of Herrera et al, in which the HLSCs prevented liver injury by modulating the expression of cytokines with liver regenerative properties [27].

As we all know, implantation is critical for stem cell therapy [27], so we made a tracer experiment after HLSC transplantation into normal and liver-damaged mice. The results showed that the labeled HLSC transplanted from the tail vein could be colonized into the liver of mice with liver injury, but could not be colonized into the liver of normal mice. However, the labeled HLSC transplanted by intraperitoneal were not found in host liver with or without injury (Additional file 1: Figure S2). In addition, to identify whether there are any long-term protective effect after HSLC/ConA due-administration, We gave the mice a second injury 50 hours later. Interestingly, the recipients were still protected by donor cells (Additional file 1: Figure S3). These evidences indicate that HYX1 cells protect against liver injury by paracrine rather than implantation.

To further study the mechanisms of HYX1 cells against ConA-induced acute liver injury, we evaluated the levels of MDSCs and CD4 ${ }^{+} \mathrm{T}$ cells in the liver and spleen of the different groups. It has been reported that MDSCs could limit tissue injury during acute hepatitis and regulate immune responses by preventing $\mathrm{T}$ cell function $[17,28]$. On the other hand, $C D 4^{+} \mathrm{T}$ cells have been confirmed to increase the ConA-induced acute 
liver injury $[19,20]$. In our study, HYX1 cells significantly increased the presence of MDSCs and decreased the levels of $\mathrm{CD} 4^{+} \mathrm{T}$ cells in the spleen and infiltrated in the liver. These results demonstrate that HYX1 cells can inhibit ConA-induced acute liver injury through the regulation of MDSCs and CD4 $4^{+} \mathrm{T}$ cells.

The expression of inflammatory cytokines in liver tissues, such as TNF- $\alpha$, IFN- $\gamma$, and IL-10, was also detected. Our results indicated that the protective role of HYX1 cell treatment in ConA-induced ALF is also associated with the reduction of TNF- $\alpha$ and IFN- $\gamma$ and increase of $I L-10$ levels.

\section{Conclusions}

In conclusion, this study demonstrated the efficacy of HLSCs in the prevention of the ConA-induced acute liver injury through the modulation of MDSCs and $\mathrm{CD}^{+} \mathrm{T}$ cell migration and cytokine secretion. Our study provides support for the use of HLSCs for the treatment of ALF.

\section{Additional file}

Additional file 1: Biochemical evaluation in the serum and the detection of tracing of transplanted cells. Supplementary data: supplementary materials and methods and supplementary

figures. Supplementary Figure 1: Biochemical evaluation of (a) ALT, (b) AST, and (c) TBIL in the serum from groups of normal, M (ConA) and $T(H Y X 1+C o n A)$ mice. Supplementary Figure 2: Tracing of transplanted cells. Supplementary Figure 3: Long-term protective effect of HYX1 on a 2nd ConA-induced liver injury. Supplementary Figure 4: Biochemical evaluation of ALT, AST and TBIL in the serum of mice with HYX1 transplantation alone. (ZIP 27721 kb)

\section{Abbreviations}

AFP: Alpha fetoprotein; ALB: Albumin; ALF: Acute liver failure; ALT: Alanine transaminase; AST: Aspartate aminotransferase; BCA: Bicinchoninic acid; CK: Cytokeratin; ConA: Concanavalin A; DMEM: Dulbecco's modified Eagle medium; DMSO: Dimethyl sulfoxide; ECL: Enhanced chemiluminescence; ELISA: Enzyme-linked immunosorbent assay; FBS: Foetal bovine serum; H\&E: Haematoxylin and eosin; HGF: Hepatocyte growth factor; HLSCs: Human liver stem cells; HYX1: Human liver stem cells 1; ICG: Indocyanine green; IFNү: Interferon-ү; IL-10: Interleukin-10; IP: Intraperitoneal; LSCs: Liver stem cells; MDSCs: Myeloid-derived suppressor cells; M-MLV: Moloney murine leukaemia virus reverse transcriptase; PAS: Periodic acid-Schiff; PBS: Phosphate-buffered saline; PVDF: Polyvinylidene difluoride; qPCR: Quantitative PCR; RT-

PCR: Reverse transcription-polymerase chain reaction; SD: Standard deviation; SDS-PAGE: Sodium dodecyl sulfate-polyacrylamide gel electrophoresis;

TBIL: Total bilirubin; TEM: Transmission electron microscopy; TNF-a: Tumour necrosis factor-a; Treg: Regulatory T cells

\section{Acknowledgements}

We thank Elsevier Language Editing Services for English editing.

\section{Funding}

This study was supported by funding from the Natural Science Foundation of China (No. 81170395, 81570556 and 81601426), National Key R\&D Program of China (No.2017YFA0103000), National Science and Technology Key Project on "Major Infectious Diseases such as HIV/AIDS, Viral Hepatitis Preventon and Treatment" (No.2017ZX10203201-005), Natural Science Foundation of Jilin Province (No.20180101137JC and 20180101130JC), and the Health Technology Innovation Project of Jilin Province (No. 2016J043).

\section{Availability of data and materials}

The datasets used and/or analysed during the current study are available from the corresponding author on reasonable request.

\section{Authors' contributions}

$Y B$ and $J L$ contributed to the design of this study, mainly performed the whole experiment, and wrote the manuscript. YY, QW, QW, XZ, and GD helped the cell culture and animal experiment. YW, YC, ZD, and ZS collected the in vivo data and contributed to the data analysis. TL contributed to the design of this study. YC, KZ and FH designed the study and edited the final manuscript. All authors read and approved the final manuscript.

\section{Ethics approval and consent to participate}

This study was reviewed and approved by the Ethics Committee of Beijing Youan Hospital, Captial Medical University.

\section{Consent for publication}

All authors approved the publication of this manuscript.

\section{Competing interests}

The authors declare that they have no competing interests.

\section{Publisher's Note}

Springer Nature remains neutral with regard to jurisdictional claims in published maps and institutional affiliations.

\section{Author details}

'Beijing Artificial Liver Treatment \& Training Center, Beijing Youan Hospital, Captial Medical University, Beijing 100069, People's Republic of China. ${ }^{2}$ Department of General Surgery, The Second Hospital of Jilin University, Changchun 130041, People's Republic of China. ${ }^{3}$ Institute of Liver Diseases, Affiliated Hospital of Jining Medical University, Jining 272067, People's Republic of China. ${ }^{4}$ Department of Neuromuscular Disease, The Third Hospital of Hebei Medical University, Shijiazhuang, People's Republic of China. ${ }^{5}$ Institute of Immunology and Molecular Medicine, Jining Medical University, Jining, People's Republic of China. ${ }^{6}$ Shanghai Meifeng Biotechnology Co., Ltd, Shanghai, People's Republic of China.

Received: 27 June 2018 Revised: 24 December 2018 Accepted: 27 December 2018 Published online: 11 January 2019

\section{References}

1. Woolbright $\mathrm{BL}$, Jaeschke $\mathrm{H}$. Role of the inflammasome in acetaminopheninduced liver injury and acute liver failure. J Hepatol. 2017;66:836-48.

2. Mu M, Zhang Z, Cheng Y, Liu G, Chen X, Wu X, Zhuang C, Liu B, Kong X, You S. Augmenter of liver regeneration (ALR) restrains concanavalin A-induced hepatitis in mice. Int Immunopharmacol. 2016;35:280-6.

3. Wang FS, Fan JG, Zhang Z, Gao B, Wang HY. The global burden of liver disease: the major impact of China. Hepatology. 2014;60:2099-108.

4. Bernal W, Wendon J. Acute liver failure. N Engl J Med. 2013;369:2525-34

5. Zhang YM, Liu ZR, Cui ZL, Yang C, Yang L, Li Y, Shen ZY. Interleukin-22 contributes to liver regeneration in mice with concanavalin A-induced hepatitis after hepatectomy. World J Gastroenterol. 2016;22:2081-91.

6. Li S, Xia Y, Chen K, Li J, Liu T, Wang F, Lu J, Zhou Y, Guo C. Epigallocatechin3-gallate attenuates apoptosis and autophagy in concanavalin A-induced hepatitis by inhibiting BNIP3. Drug Des Devel Ther. 2016;10:631-47.

7. Wang HX, Liu M, Weng SY, Li JJ, Xie C, He HL, Guan W, Yuan YS, Gao J. Immune mechanisms of concanavalin A model of autoimmune hepatitis. World J Gastroenterol. 2012;18:119-25.

8. Shen M, Lu J, Cheng P, Lin C, Dai W, Wang F, Wang C, Zhang Y, Chen K, $\mathrm{Xu} L$, et al. Ethyl pyruvate pretreatment attenuates concanavalin A-induced autoimmune hepatitis in mice. PLoS One. 2012;9:e87977.

9. Sapisochin G, Bruix J. Liver transplantation for hepatocellular carcinoma: outcomes and novel surgical approaches. Nat Rev Gastroenterol Hepatol. 2017;14:203-17.

10. Zheng P, Chang X, Lu Q, Liu Y. Cytopenia and autoimmune diseases: a vicious cycle fueled by mTOR dysregulation in hematopoietic stem cells. J Autoimmun. 2013;41:182-7.

11. Ra JC, Kang SK, Shin IS, Park HG, Joo SA, Kim JG, Kang BC, Lee YS, Nakama $K$, Piao $M$, et al. Stem cell treatment for patients with autoimmune disease 
by systemic infusion of culture-expanded autologous adipose tissue derived mesenchymal stem cells. J Transl Med. 2011;9:181.

12. Kuijk EW, Rasmussen S, Blokzijl F, Huch M, Gehart H, Toonen P, Begthel H, Clevers H, Geurts AM, Cuppen E. Generation and characterization of rat liver stem cell lines and their engraftment in a rat model of liver failure. Sci Rep. 2016;6:22154.

13. Esrefoglu M. Role of stem cells in repair of liver injury: experimental and clinical benefit of transferred stem cells on liver failure. World J Gastroenterol. 2013;19:6757-73.

14. Wang Y, Yu X, Chen E, Li L. Liver-derived human mesenchymal stem cells: a novel therapeutic source for liver diseases. Stem Cell Res Ther. 2016;7:71

15. Tao $\mathrm{Y}-\mathrm{C}$, Wang $\mathrm{M}-\mathrm{L}$, Chen $\mathrm{E}-\mathrm{Q}$, Tang H. Stem cells transplantation in the treatment of patients with liver failure. Curr Stem Cell Res Ther. 2018;13: 193-201.

16. Avia W, Julian K, Alex YH, Mei Z. Nanoparticle systems modulating myeloidderived suppressor cells for cancer immunotherapy. Curr Top Med Chem. 2017;17:1843-57.

17. Hammerich L, Tacke F. Emerging roles of myeloid derived suppressor cells in hepatic inflammation and fibrosis. World J Gastrointest Pathophysiol. 2015;6:43-50

18. Kondo $Y$, Shimosegawa $T$. Significant roles of regulatory $T$ cells and myeloid derived suppressor cells in hepatitis B virus persistent infection and hepatitis B virus-related HCCs. Int J Mol Sci. 2015;16:3307-22.

19. Hu B, Zou Y, Liu S, Wang J, Zhu J, Li J, Bo L, Deng X. Salidroside attenuates concanavalin A-induced hepatitis via modulating cytokines secretion and lymphocyte migration in mice. Mediat Inflamm. 2014;2014:314081.

20. Ramirez FMA, Silva A, Mason D. Glucocorticoids induce the expression of CD8 alpha chains on concanavalin A-activated rat CD4+ T cells: induction is inhibited by rat recombinant interleukin 4. J Exp Med. 1992;176:1551-9.

21. Bi YZ, Fan Z, Chen DF, Li SS, Wang QY, Gao PF, Wang QQ, Duan ZP, Chen Y, Kong $L B$, et al. Protective effect of intraperitoneal transplantation of human liver-derived stem cells at different times against concanavalin A-induced acute liver injury in mice. Zhonghua Gan Zang Bing Za Zhi. 2017;25:205-10.

22. Soares PA, Nascimento CO, Porto TS, Correia MT, Porto AL, Carneiro-daCunha MG. Purification of a lectin from Canavalia ensiformis using PEGcitrate aqueous two-phase system. J Chromatogr B Anal Technol Biomed Life Sci. 2011:879:457-60.

23. Hong F, Jaruga B, Kim WH, Radaeva S, El-Assal ON, Tian Z, Nguyen VA, Gao B. Opposing roles of STAT1 and STAT3 in T cell-mediated hepatitis: regulation by SOCS. J Clin Invest. 2002;110:1503-13.

24. Cheng P, Chen K, Xia Y, Dai W, Wang F, Shen M, Wang C, Yang J, Zhu R, Zhang $\mathrm{H}$, et al. Hydrogen sulfide, a potential novel drug, attenuates concanavalin A-induced hepatitis. Drug Des Devel Ther. 2014;8:1277-86.

25. Yang $Q$, Wang J, Liu R, Wang Z, Li Y, Zhang Y, Hao X, Huang Y, Xie W, Wei $\mathrm{H}$. Amelioration of concanavalin A-induced autoimmune hepatitis by magnesium isoglycyrrhizinate through inhibition of CD4(+)CD25(-)CD69(+) subset proliferation. Drug Des Devel Ther. 2016;10:443-53.

26. Yang T, Wang Y, Jiang S, Liu X, Yu Z. Hepatocyte growth factor-induced differentiation of bone mesenchymal stem cells toward hepatocyte-like cells occurs through nuclear factor-kappa B signaling in vitro. Cell Biol Int. 2016; 40:1017-23.

27. Herrera MB, Fonsato V, Bruno S, Grange C, Gilbo N, Romagnoli R, Tetta C, Camussi G. Human liver stem cells improve liver injury in a model of fulminant liver failure. Hepatology. 2013;57:311-9.

28. Youn Jl, Gabrilovich DI. The biology of myeloid-derived suppressor cells: the blessing and the curse of morphological and functional heterogeneity. Eur J Immunol. 2010:40:2969-75.

Ready to submit your research? Choose BMC and benefit from:

- fast, convenient online submission

- thorough peer review by experienced researchers in your field

- rapid publication on acceptance

- support for research data, including large and complex data types

- gold Open Access which fosters wider collaboration and increased citations

- maximum visibility for your research: over $100 \mathrm{M}$ website views per year

At BMC, research is always in progress.

Learn more biomedcentral.com/submissions 